\title{
A Twelve-Ports Dual-Polarized MIMO Log-Periodic Dipole Array Antenna for UWB Applications
}

\author{
Li-yan CHEN, Jing-song HONG, Muhammad AMIN \\ School of Physical Electronics, University of Electronic Science and Technology of China, 610054 Chengdu, China \\ 727683316@qq.com,cemlab@uestc.edu.cn, aminssphysics@hotmail.com \\ Submitted August 26, 2018 / Accepted December 12, 2018
}

\begin{abstract}
A twelve-port dual-polarized multiple-inputmultiple-output (MIMO) log-periodic dipole array (LPDA) antenna for ultra-wideband (UWB) applications is proposed in this paper. In the MIMO antenna, there are 12 antennas, six for horizontal polarization and six for vertical polarization. In order to achieve dual linear polarizations and beam switching, six horizontal antennas are placed in a sequential, rotating arrangement on a horizontal substrate panel with an equal inclination angle of $60^{\circ}$ to form a symmetrical structure, while the other six antennas for vertical polarization are inserted through slots made on the horizontal substrate panel. In addition, all twelve antennas share a hexagon common ground on the back of substrate and feed by micro-strip line. A prototype of the MIMO antenna is manufactured and the reflection coefficients, coupling isolation, radiation pattern and peak gain are measured. The MIMO performance of the MIMO LPDA antenna is predicted and evaluated by envelope correlation coefficient (ECC) and total active reflection coefficient (TARC).
\end{abstract}

\section{Keywords}

UWB, MIMO, LPDA

\section{Introduction}

Ultra-wideband (UWB) applications have remained one of the most rapidly developing technologies in wireless applications, since the Federal Communication Commission (FCC) has assigned an unlicensed 3.1-10.6 GHz bandwidth. The Multiple-Input-Multiple-Output (MIMO) technology has incomparable advantages in improving the wireless link transmission capacity and reliability. Combining MIMO technology with UWB technology is an efficient way to decrease multipath fading in UWB systems [1].

Researchers have proposed various decoupling methods in the recent years [2-7], such as using electromagnetic band-gap (EBG) structure [2], placing a tree-shaped parasitic structure on the antenna ground [3], using a T-shaped protruded ground stub [4] to minimize the mutual coupling between radiating elements, a complementary split-ring resonator (CSRR) etching on the antenna ground [5]. The antenna in [6] does not have any decoupling structure; the high isolation performance has been achieved by the asymmetrical and complementary structures of the quasi-selfcomplementary antenna (QSCA).

Some MIMO antenna arrays have been presented in last few years [7-10]. In [7] and [8], a three port MIMO Yagi-Uda antenna array with integrated balun structure and a novel six-port MIMO array antenna with high port isolation have been proposed. These two arrays were used for WLAN system, more importantly, these arrays could not support beam-switching and dual-polarization. On the other hand, two twelve-port dual-band dual-polarized MIMO array antennas were proposed in [9] and [10], respectively. Although, these two array antennas could support beam switching and dual-polarization, but could not be applied to the UWB systems.

In this paper, a twelve-port dual-polarized MIMO LPDA antenna for UWB applications in a size of $240 \times(150+45 \sqrt{3}) \times 45 \mathrm{~mm}^{3}$ is proposed. The design process and geometry of the proposed MIMO LPDA antenna is illustrated in Fig. 1 and Fig. 2, respectively. First, six horizontal LPDAs (H1-H6) are placed in rotating arrangement with an equal angle of $60^{\circ}$ on the top and bottom face of an FR4 substrate to encircle the hexagon ground. Then, other six vertical LPDAs (V1-V6) are inserted into the corresponding slot etched on the diagonal of the substrate. Besides, all twelve antennas share a hexagon common ground on the back of substrate and feed by micro-strip line. Compared to the previous MIMO array antennas in [8-11], this MIMO LPDA antenna has the superiority of a novel antenna structure, UWB band, higher isolation, higher antenna gain and better MIMO performance.

\section{Antenna Design}

\subsection{LPDA Antenna Element}

In this MIMO LPDA antenna, LPDA antenna is chosen as the antenna element due to its advantage of high gain and directional radiation pattern. Figure 3 shows the geometry of LPDA element. The LPDA antenna consists of 
13 different dipoles named as arm 1, arm 2, arm 3... arm 13 respectively, which are printed on top and bottom side of a $75 \times 45 \times 1.6 \mathrm{~mm}^{3}$ FR4 (loss tangent of 0.02 and dielectric constant of 4.4) substrate and fed by micro-strip line.

First, the number of dipole $N$, the spacing factor $\sigma$, the scale factor $\tau$ are three key parameters in the design process of a $\log$-periodic antenna, where $\sigma=0.14, \tau=0.85$ and $N=13$ are selected in the proposed LPDA.

Second, the first resonance frequency $f_{l}$ of log-periodic dipole antenna depends on the length of the longest dipole and could be calculated by the formula (1) and (2) [10],

$$
\begin{gathered}
f_{1}=\frac{c}{4 L_{\text {max }} \sqrt{\varepsilon_{\text {eff }}}}, \\
\varepsilon_{\text {eff }}=\frac{\varepsilon_{\mathrm{r}}+1}{2}+\frac{\varepsilon_{\mathrm{r}}-1}{2} \frac{1}{\sqrt{1+\frac{12 h}{W_{\text {max }}}}}
\end{gathered}
$$

where $c$ is the speed of light, $L_{\max }$ is the length of the longest dipole and $\varepsilon_{\mathrm{r}}$ is the relative dielectric constant of the substrate, $h$ is the height of substrate and $W_{\max }=3.6 \mathrm{~mm}$ is the width of the longest dipole. To obtain the UWB characteristic, the first resonance frequency $f_{1}$ is chosen as $3 \mathrm{GHz}$. Then, on the basis of (1) and (2), the calculated $L_{\max }$ is $18 \mathrm{~mm}$ approximately.

Finally, after confirming the length and width of the longest dipole, the dimensions of remainder dipoles and the distance between the two adjacent dipoles can be computed according to the scale formula (3) and (4) [10],

$$
\begin{gathered}
\frac{L_{n+1}}{L_{n}}=\frac{W_{n+1}}{W_{n}}=\tau, \\
\frac{d_{n}}{L_{n}}=4 \sigma
\end{gathered}
$$

where $n=1,2,3, \ldots, 13, L_{\max }=L_{1}, W_{\max }=W_{1}$. The ultimate parameters of the LPDA antenna element are illustrated in Tab. 1.
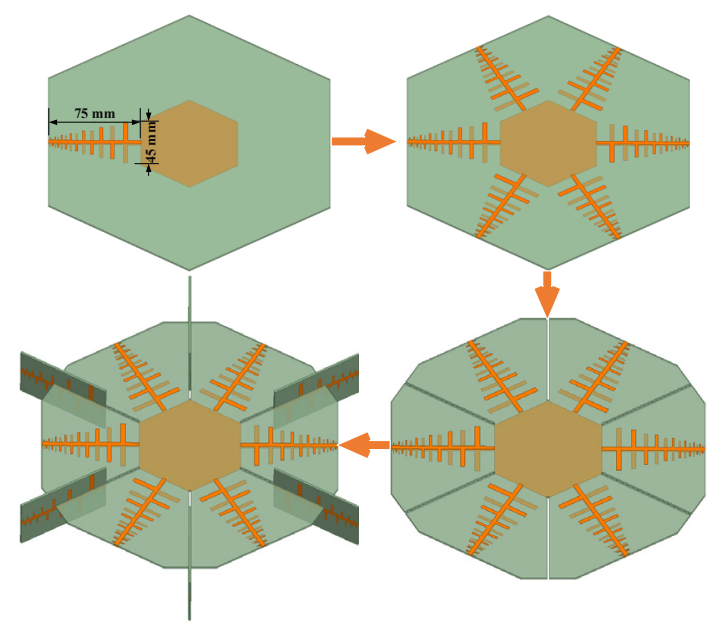

Fig. 1. The design process of the proposed MIMO LPDA antenna.

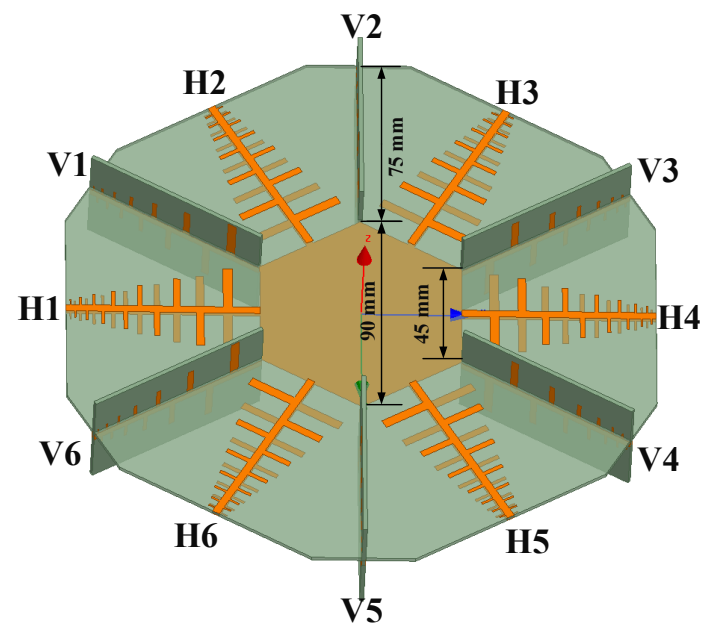

Fig. 2. The geometry of the proposed twelve-port (port H1 H6, V1 V6) MIMO LPDA antenna.

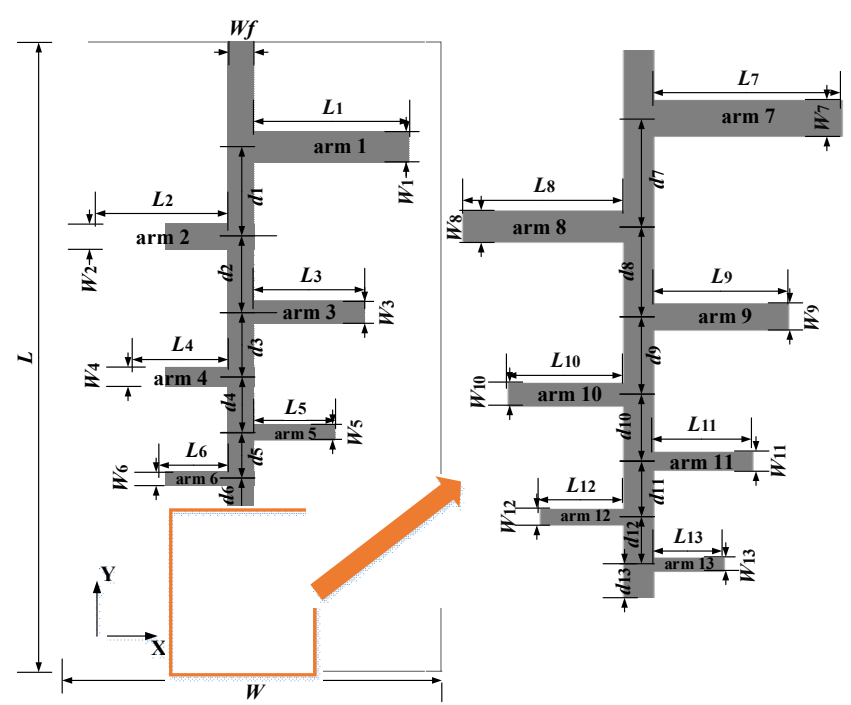

Fig. 3. The geometry of LPDA antenna element.

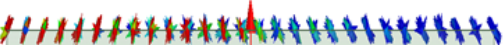

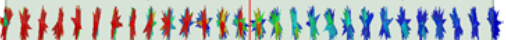

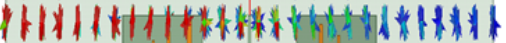

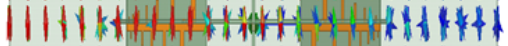

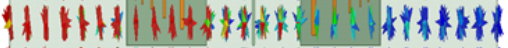

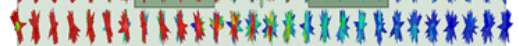

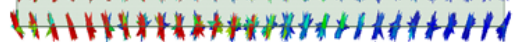

(a)

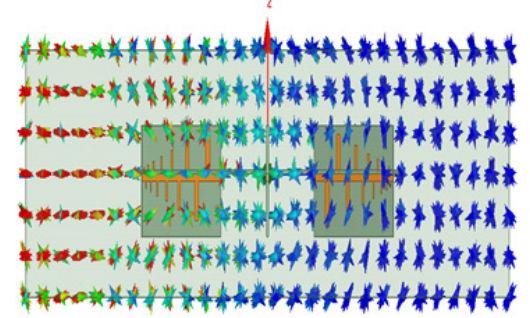

(b)

Fig. 4. Simulated E-field distribution of the MIMO LPDA antenna at $4 \mathrm{GHz}$ in the far field when port (a) $\mathrm{H} 1$ and (b) V1 is excited, respectively. 


\begin{tabular}{|c|c|c|c|c|c|c|c|c|c|c|c|c|c|c|}
\hline $\boldsymbol{L}_{\mathbf{1}}$ & $\boldsymbol{L}_{\mathbf{2}}$ & $\boldsymbol{L}_{\mathbf{3}}$ & $\boldsymbol{L}_{\mathbf{4}}$ & $\boldsymbol{L}_{\mathbf{5}}$ & $\boldsymbol{L}_{\mathbf{6}}$ & $\boldsymbol{L}_{7}$ & $\boldsymbol{L}_{\mathbf{8}}$ & $\boldsymbol{L}_{\mathbf{9}}$ & $\boldsymbol{L}_{\mathbf{1 0}}$ & $\boldsymbol{L}_{\mathbf{1 1}}$ & $\boldsymbol{L}_{\mathbf{1 2}}$ & $\boldsymbol{L}_{\mathbf{1 3}}$ & $\boldsymbol{W}_{\mathbf{1}}$ & $\boldsymbol{W}_{\mathbf{2}}$ \\
\hline 18.5 & 15.8 & 13.2 & 11.4 & 9.7 & 8.2 & 6.9 & 5.9 & 5.1 & 4.3 & 3.6 & 3.1 & 2.6 & 3.6 & 3.0 \\
\hline $\boldsymbol{W}_{\mathbf{3}}$ & $\boldsymbol{W}_{\mathbf{4}}$ & $\boldsymbol{W}_{\mathbf{5}}$ & $\boldsymbol{W}_{\mathbf{6}}$ & $\boldsymbol{W}_{\mathbf{7}}$ & $\boldsymbol{W}_{\mathbf{8}}$ & $\boldsymbol{W}_{\mathbf{9}}$ & $\boldsymbol{W}_{\mathbf{1 0}}$ & $\boldsymbol{W}_{\mathbf{1 1}}$ & $\boldsymbol{W}_{\mathbf{1 2}}$ & $\boldsymbol{W}_{\mathbf{1 3}}$ & $\boldsymbol{d}_{\mathbf{1}}$ & $\boldsymbol{d}_{\mathbf{2}}$ & $\boldsymbol{d}_{\mathbf{3}}$ & $\boldsymbol{d}_{\mathbf{4}}$ \\
\hline 2.6 & 2.2 & 1.8 & 1.6 & 1.3 & 1.1 & 0.9 & 0.8 & 0.7 & 0.6 & 0.5 & 10.4 & 8.8 & 7.5 & 6.4 \\
\hline $\boldsymbol{d}_{\mathbf{5}}$ & $\boldsymbol{d}_{\mathbf{6}}$ & $\boldsymbol{d}_{7}$ & $\boldsymbol{d}_{\mathbf{8}}$ & $\boldsymbol{d}_{\mathbf{9}}$ & $\boldsymbol{d}_{\mathbf{1 0}}$ & $\boldsymbol{d}_{\mathbf{1 1}}$ & $\boldsymbol{d}_{\mathbf{1 2}}$ & $\boldsymbol{d}_{\mathbf{1 3}}$ & $\boldsymbol{L}$ & $\boldsymbol{W}$ & $\boldsymbol{H}$ & $\boldsymbol{W f}$ & & \\
\hline 5.4 & 4.6 & 3.9 & 3.3 & 2.8 & 2.4 & 2 & 1.7 & 1.5 & 75 & 45 & 1.6 & 3 & & \\
\hline
\end{tabular}

Tab. 1. The ultimate parameters of the LPDA antenna element, all parameters are in $\mathrm{mm}$.

\subsection{Dual-Polarized Characteristic}

The dual-polarized characteristics of the proposed MIMO LPDA antenna are obtained by the orthogonal arrangement between horizontal LPDA element and vertical LPDA element. In other words, any two perpendicular LPDA elements produce an orthogonal current on the MIMO array, which results in dual-polarized electric field in the far field.

Figure 4 illustrates the simulated electric field distributions at $4 \mathrm{GHz}$ in the far field region when port $\mathrm{H} 1$ and $\mathrm{V} 1$ are excited, respectively. Figure 4 shows that main electric field is vertical polarized when $\mathrm{H} 1$ is excited. However, when V1 is excited, most electric field transforms to horizontal polarized, as shown in Fig. 4, which is consonant with the predicted electric field in the far field region.

\subsection{Beam Switching Characteristic}

Beam switching characteristics is the particularity of the proposed MIMO LPDA antenna. Figure 5 shows simulated $3 \mathrm{D}$ radiation pattern of the MIMO LPDA antenna at $4 \mathrm{GHz}$ when port $\mathrm{H} 1, \mathrm{~V} 2, \mathrm{H} 4$ and V5 is excited, respectively. It is shown in Fig. 5(a) when port H1 is excited, the radiation beam is directed towards $-\mathrm{y}$ direction in xoy plane along the H1 LPDA element. On the other hand, when port $\mathrm{H} 4$ is excited, the radiation beam transforms to the $+y$ direction, along with the H4 LPDA element, see Fig. 5(b). Namely, the radiation beam shifts to the corresponding direction when different ports are excited. Thus, the presented MIMO LPDA antenna using LPDA as an antenna element has twelve fixed beams for twelve directions could be selected to decrease the interference by turning on/off the corresponding port, which is an effective way to overcome the mitigate interference from other wireless systems.

\section{Measured Results and Discussion}

\subsection{S-parameters}

The proposed MIMO LPDA antenna have been fabricated and the S-parameters of the proposed antenna measured with Field Fox N9918A microwave network analyzer to verify predicted characteristics, employing a coaxial cable at the desired antenna port and connecting the others to $50 \Omega$ loads, as illustrated in Fig. 6. Figure 7 shows measured $S_{11}$ of the MIMO LPDA antenna when port H1 H6, $\mathrm{V} 1 \sim \mathrm{V} 6$ is measured, respectively. Measured results indi- cate that all LPDA elements operate from $2.8 \mathrm{GHz}$ to $11 \mathrm{GHz}$ with $S_{11}<-15 \mathrm{~dB}$. Some discrepancies can be seen in $S_{11}$ curves when different ports are measured, which may be caused by the different manufacture tolerance and SMA connectors.

The isolation between random two LPDA elements of the MIMO LPDA antenna can be efficiently illustrated by $S_{\mathrm{H} 1 \mathrm{H} 2}, S_{\mathrm{H} 1 \mathrm{H} 3}, S_{\mathrm{H} 1 \mathrm{H} 4}, S_{\mathrm{H} 1 \mathrm{~V} 1}, S_{\mathrm{H} 1 \mathrm{~V} 2}, S_{\mathrm{H} 1 \mathrm{~V} 3}, S_{\mathrm{V} 1 \mathrm{~V} 2}, S_{\mathrm{V} 1 \mathrm{~V} 3}$ and $S_{\mathrm{V} 1 \mathrm{~V} 4}$ due to the symmetry structure of the MIMO antenna. Figure 8 shows that the isolation between the two random LPDA elements is more than $40 \mathrm{~dB}$ in the operating frequency band. In some low-frequency spectrum, the isolation is less than $40 \mathrm{~dB}$ but more than $30 \mathrm{~dB}$.

The radiation performance of the MIMO LPDA antenna is measured in the one of the largest anechoic chamber in China, as shown in Fig. 9. The radiation characteristics of the MIMO antenna are obtained by the two-axis dual-polarization pattern measurement system. In this section, only the results of one horizontal antenna (H1) and one vertical antenna (V1) are reported since the array has

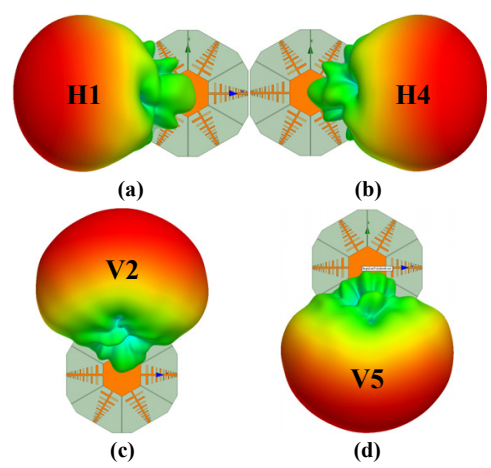

Fig. 5. Simulated $3 D$ radiation pattern of the MIMO LPDA antenna at $4 \mathrm{GHz}$ when port $\mathrm{H} 1, \mathrm{~V} 2, \mathrm{H} 4$ and V5 are excited, respectively.

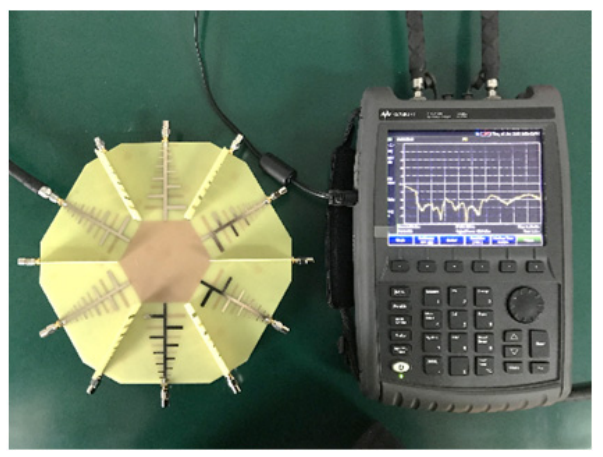

Fig. 6. Photograph of the MIMO LPDA antenna when port H1 is measured with Field Fox N9918A microwave network analyzer. 

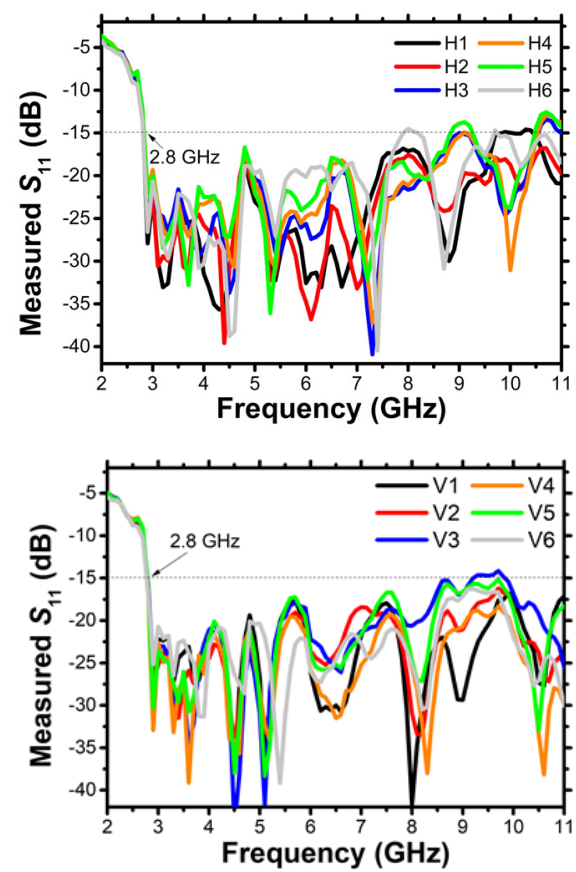

Fig. 7. Measured $S_{11}$ of MIMO LPDA antenna when port $\mathrm{H} 1 \sim \mathrm{H} 6, \mathrm{~V} 1 \sim \mathrm{V} 6$ are excited, respectively.
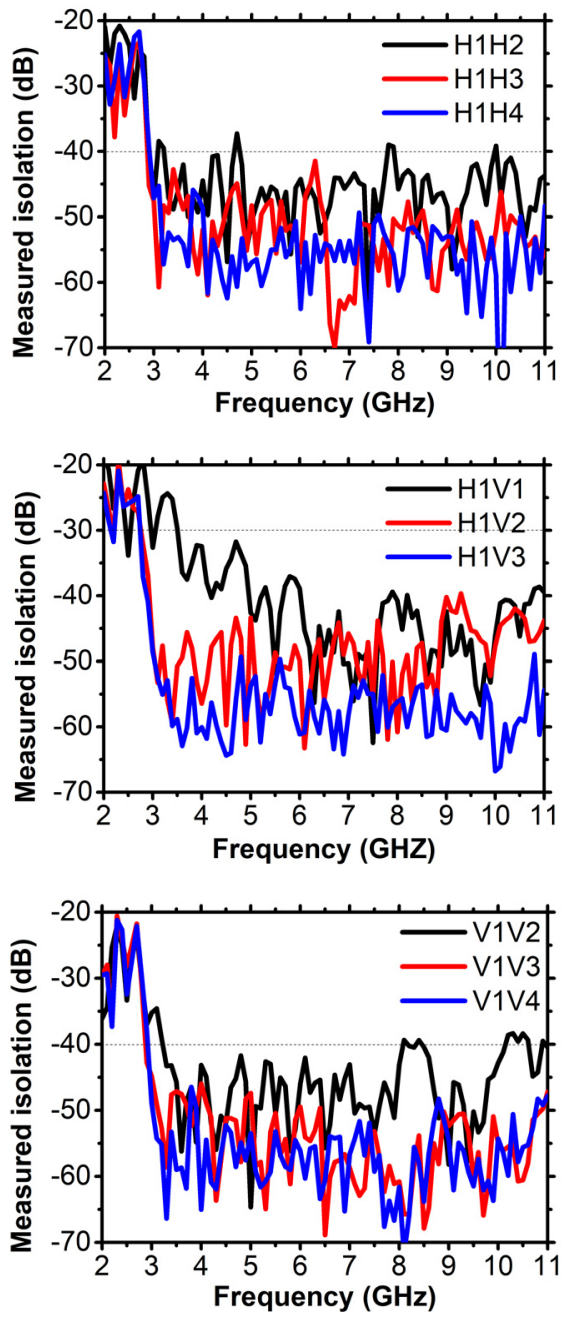

Fig. 8. Measured isolation between random two LPDA elements of the MIMO LPDA antenna. asymmetrical arrangement. Figure 10 illustrates simulated and measured radiation patterns (yoz-plane and xoy-plane) of the MIMO LPDA antenna. The co-polarization in both plane are pointing to $-\mathrm{y}$-axis $\left(270^{\circ}\right)$ when ports $\mathrm{H} 1$ and $\mathrm{V} 1$ is measured, respectively, as shown in Fig. 10 (a, b, c). But in xoy-plane the co-polarization is pointing to $300^{\circ}$ when the port V1 is measured. The radiation beam is directed along the V1 LPDA element, as shown in V1 in Fig. 10 (d, e, f), which verified the beam-switching characteristics of the proposed design. The x-polarization in both planes is growing worse with the frequency increase, but not more than $-15 \mathrm{~dB}$. Figure 11 illustrates the measured peak gain of the proposed MIMO LPDA antenna when ports $\mathrm{H} 1$ or $\mathrm{V} 1$ is measured, the average peak gain in the operating UWB band is $7.17 \mathrm{dBi}$ and $7.42 \mathrm{dBi}$, respectively.

\subsection{MIMO Performance}

The value of ECC represents how the two antennas are coupled to each other. For achieving good channel characteristics and antenna performance, the ECC must be less than 0.05 . For a uniformly multipath environment, the ECC between random two LPDA elements can be calculated from the S-parameters (5) [11],

$$
E C C_{i j}=\left|\frac{\left|S_{i i}^{*} S_{i j}+S_{j i}^{*} S_{j j}\right|}{\left|\left(1-\left|S_{i i}\right|^{2}-\left|S_{i j}\right|^{2}\right)\left(1-\left|S_{j i}\right|^{2}-\left|S_{j j}\right|^{2}\right) \eta_{\text {radi }} \eta_{\text {radj }}\right|^{\frac{1}{2}}}\right|^{2}
$$

The ECC of this MIMO LPDA antenna are sufficiently indicated by LPDA $\mathrm{H} 1$ and $\mathrm{H} 2, \mathrm{H} 1$ and V1, V1 and V2 due to the symmetric antenna structure. Figure 12 shows the measured ECC of all horizontal and vertical LPDA elements. Results show that the measured ECC is approximately zero from $3 \mathrm{GHz}$ to $11 \mathrm{GHz}$.

For MIMO antenna systems, traditional scattering matrix cannot be sufficient to predict the real antenna performance. TARC which takes coupling effect into account has been proposed. In this 12-ports MIMO antenna system, TARC can be calculated from S-parameters (6) [12],

$$
\text { TARC }=\sqrt{\left(\sum_{i=1}^{12}\left(\sum_{k=1}^{12} S_{i k}\right)^{2}\right) / 12} .
$$

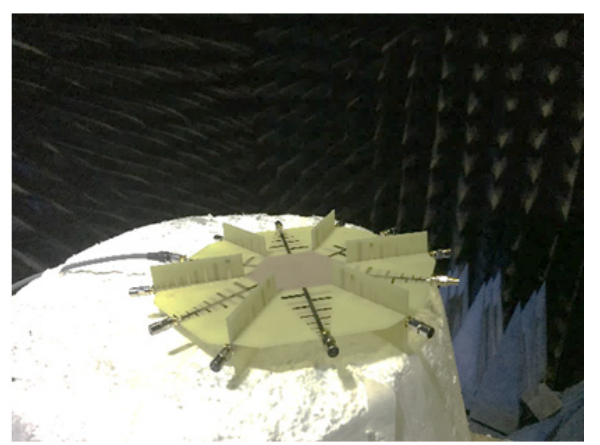

Fig. 9. Photograph of the MIMO LPDA antenna mounted on measurement system. 

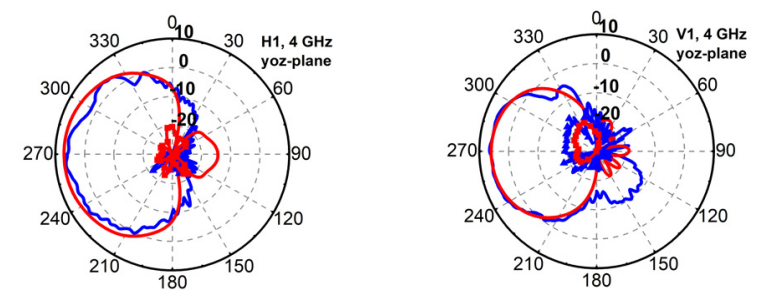

(a)
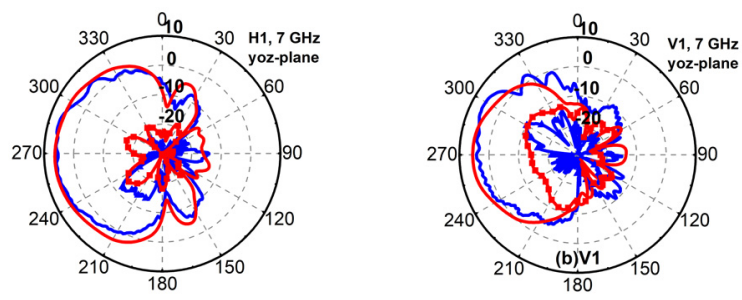

(b)
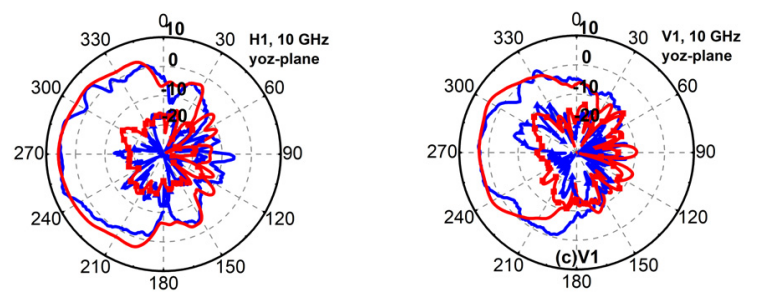

(c)
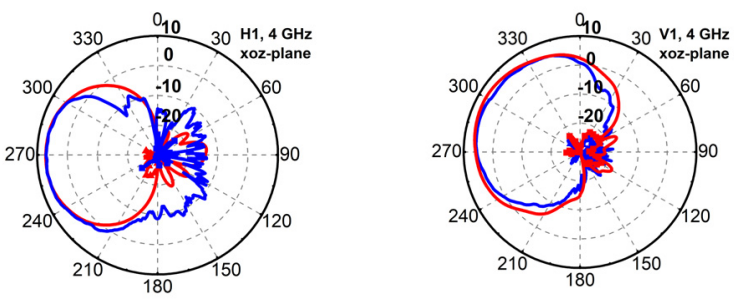

(d)
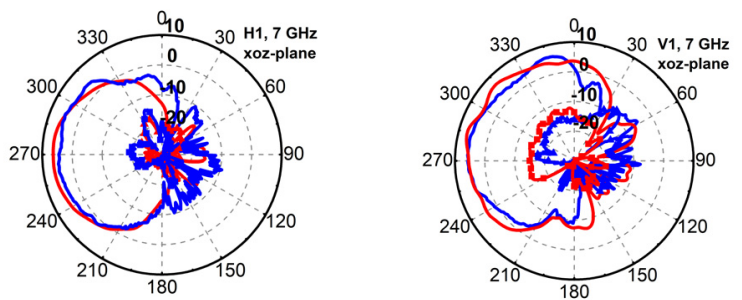

(e)
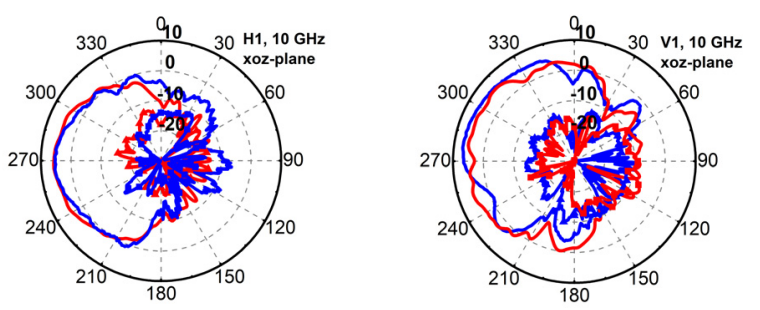

(f)

Measured co-pol $\longrightarrow$ - Measured $\mathrm{x}-\mathrm{pol}$

Fig. 10. Simulated and measured radiation pattern of MIMO LPDA antenna.

The TARC of the MIMO LPDA antenna is less than $-25 \mathrm{~dB}$ for the UWB band, as illustrated in Fig. 12.

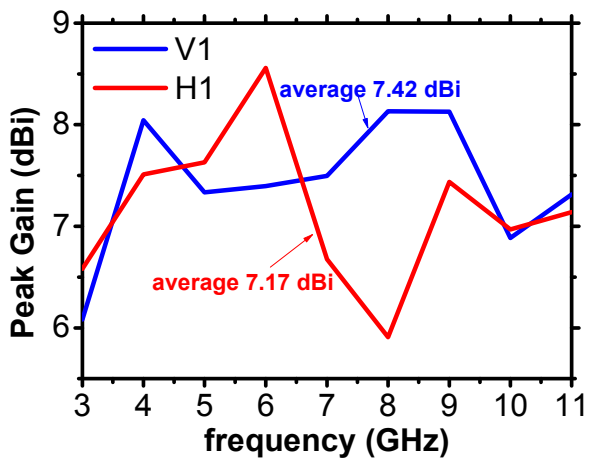

Fig. 11. Measured peak gain of the MIMO LPDA antenna.

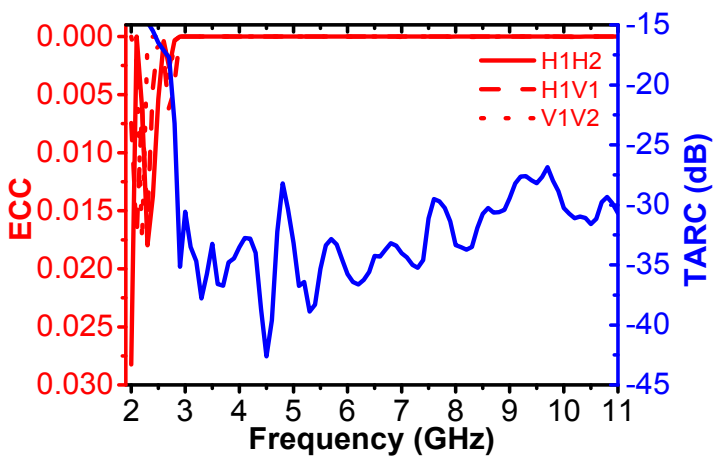

Fig. 12. Measured ECC and TARC of the MIMO LPDA antenna.

\section{Conclusion}

In this paper, a twelve-port dual-polarized MIMO LPDA antenna for UWB applications in a size of $240 \times(150+45 \sqrt{3}) \times 45 \mathrm{~mm}^{3}$ is presented. To obtain high isolation, beam switching and dual-polarized characteristics, six horizontal LPDA elements are placed in rotating arrangement with an equal angle of $60^{\circ}$ on an FR4 substrate and other six vertical LPDA elements are inserted into the corresponding slot etched on the diagonal of horizontal plane of the same substrate. In addition, all twelve LPDA elements share a hexagon common ground on the back of the substrate and are fed by micro-strip line. The MIMO antenna was also manufactured, measured results show that the designed MIMO LPDA antenna exhibits an impedance match from $2.8-11 \mathrm{GHz}$ with nearly $S_{11}<-15 \mathrm{~dB}$, high isolation more than $30 \mathrm{~dB}$, directional radiation pattern, average peak gain of $7.245 \mathrm{dBi}$, $E C C<0.005$ and TARC $<-25 \mathrm{~dB}$ over the UWB band, which makes it a potential candidate for UWB applications. Furthermore, the proposed MIMO antenna is also a possible candidate in MIMO wireless local area network (WLAN) applications and worldwide interoperability for microwave access (WiMAX) applications.

\section{References}

[1] KAISER, T., ZHENG, F., DIMITROV, E. An overview of ultrawide-band systems with MIMO. Proceedings of the IEEE, 
2009, vol. 97, no. 2, p. 285-312. DOI: 10.1109/JPROC.2008.2008784

[2] Coulombe, M., FARZANeH, K. S., CAlOZ, C. Compact elongated mushroom (EM)-EBG structure for enhancement of patch antenna array performances. IEEE Transactions on Antennas and Propagation, 2010, vol. 58, no. 4, p. 1076-1086. DOI: 10.1109/TAP.2010.2041152

[3] ZHANG, S., YING, Z., XIONG, J., et al. Ultrawide-band $\mathrm{MIMO} /$ diversity antennas with a tree-like structure to enhance wideband isolation. IEEE Antennas Wireless and Propagation Letters, 2009, vol. 8, p. 1279-1282. DOI: 10.1109/LAWP.2009.2037027

[4] LIU LI, CHEUNG, S. W., YUK, T. I. Compact MIMO antenna for portable UWB applications with band-notched characteristic. IEEE Transactions on Antennas and Propagation, 2015, vol. 63, no. 5, p. 1917-1924. DOI: 10.1109/TAP.2015.2406892

[5] KHAN, M. S., CAPOBIANCO, A. D., ASIF, S. M., et al. A compact CSRR enabled UWB MIMO antenna. IEEE Antennas and Wireless Propagation Letters, 2009, vol. 16, p. 808-812. DOI: 10.1109/LAWP.2016.2604843

[6] LIU, X. L., WANG, Z. D., YIN, Y. Z., et al. A compact ultrawideband MIMO antenna using QSCA for high isolation. IEEE Antennas and Wireless Propagation Letters, 2014, no. 13, p. 1497-1500. DOI: 10.1109/LAWP.2014.2340395

[7] HONMA, N., SEKI, T., NISHIKAWA, K., et al. Compact sixsector antenna employing three intersecting dual-beam microstrip Yagi-Uda arrays with common director. IEEE Transactions on Antennas and Propagation, 2006, vol. 54, no. 11, p. 3055-3062. DOI: $10.1109 /$ TAP.2006.883980

[8] HAN, W. W., ZHOU, X. P., OUYANG, J., et al. A six-port MIMO antenna system with high isolation for $5 \mathrm{GHz}$ WLAN access points. IEEE Antennas and Wireless Propagation Letters, 2014, vol. 13, p. 880-883. DOI: 10.1109/LAWP.2014.2310739

[9] ZHENG, W. C., ZHANG, L., LI, Q. X., et al. Dual-band dualpolarized compact bowtie antenna array for anti-interference MIMO WALN. IEEE Transactions on Antennas and Propagation, 2014, vol. 62, no. 1, p. 237-246. DOI: 10.1109/TAP.2013.2287287

[10] LIANG, J. J., HONG, J. S., ZHAO, J. B., et al. Dual-band dualpolarized compact log-periodic dipole array for MIMO WLAN applications. IEEE Antennas and Wireless Propagation Letters, 2015, vol. 14, p. 751-754. DOI: 10.1109/LAWP.2014.2378772

[11] HALLBJORNER, P. The significance of radiation efficiencies when using S-parameters to calculate the received signal correlation from two antennas. IEEE Antennas and Wireless Propagation Letters, 2005, vol. 4, no. 1, p. 97-99. DOI: 10.1109/LAWP.2005.845913

[12] GAO, Y., CHEN, X. D., YING, Z. N., et al. Design and performance investigation of a dual-element PIFA array at 2.5 $\mathrm{GHz}$ for MIMO terminal. IEEE Transactions on Antennas and Propagation, 2007, vol. 55, no. 12, p. 3433-3441. DOI: 10.1109/TAP.2007.910353

\section{About the Authors ...}

Li-yan CHEN was born in Wuhan, China. He received his B.S. degree from Shanxi University in 2016. He is now working towards his M.S. degree in Radio Physics at the University of Electronic Science and Technology of China (UESTC). His interests include MIMO antenna and MIMO system.

Jing-song HONG received the B.S. degree in Electromagnetic from LanZhou University, China, in 1991, and the M.S. and Ph.D. degrees in Electrical Engineering from the University of Electronic Science and Technology of China (UESTC), in 2000 and 2005, respectively. He is now a professor with UESTC. His research interests include the use of numerical techniques in electromagnetic and the use of microwave methods for materials characterization.

Muhammad AMIN was born in D.I. Khan, Pakistan. He received his Master degree from Gomal University D.I. Khan Pakistan in 2003. Since September 2014 he is a Ph.D. student at the University of Electronic Science and Technology of China (UESTC). His research direction is Radio Physics. His research interests include antenna technology and wireless communication technology. 